\title{
Effect of land-use management systems on coupled physical and mechanical, chemical and biological soil processes: how can we maintain and predict soil properties and functions?
}

\author{
Rainer HORN (ه) ${ }^{1}$, Winfried E. H. BLUM ${ }^{2}$ \\ 1 Institute of Plant Nutrition and Soil Science, 24118 Kiel, Germany \\ 2 Institute of Soil Research, University of Natural Resources and Life Sciences (BOKU), 1190 Vienna, Austria
}

Soil is one of the most critical life-supporting components of the biosphere. Soil provides many ecosystem services, such as a habitat for biodiversity, water and nutrients, food, feed, fiber and energy, but also serves as archaeological repositories. In addition to natural changes in pedological properties and functions over the course of time (soil genesis), their properties also undergo intense and irreversible changes due to a non-site adjusted land management and improper application of machinery, sealing and techniques as well as chemical impacts including acidity from acid rain, heavy metals pollution and salinity.

The discrepancy between a growing population (predicted to be 9 billion people by 2050) with a consequently increasing demand for food and space and an irreversible daily soil degradation of about $400 \mathrm{~km}^{2}$ worldwide enhances the urgency for a more site-specific soil management. At the same time, the limits of actual external physical, mechanical or chemical stresses should be determined in relation to the actual internal soil strength to depth, which defines the degree of resilience. Worldwide, a diverse range of soil and land-use classification systems are available. According to the definition of Blum and Eswaran ${ }^{[1]}$, land quality classes ranges from 1 (perfect) to 9 (inappropriate) for food production or living. About $25 \%$ of the world population live on $12 \%$ of the area with perfect soils (classes $1-3$ ) and another $54 \%$ on $34 \%$ of areas with medium soil properties. However, more than $25 \%$ of the world's present population live in areas with mostly inappropriate soils (classes $7-9$ ), representing about $45 \%$ of the areas with these soils. Thus, we might face multiple problems including further migration, which can result in an overstress of originally fertile or productive soils. Consequently, an additional irreversible degradation of initially sitespecific beneficial properties and functions can be expected.

Soils as three phase systems have only a limited resilience and sustainability. Exceeding boundary conditions not only worsens chemical and biological soil properties and functions, but also reduces the accessibility of particle or pore surfaces and alters pore size and distribution. This changes the flux of water, gas and heat, and will therefore affect the actual contributions of soils as components of ecosystems. This is a problematic situation because the relevant reference magnitude is variable as it depends on the actual definition of the expected functionality of soils or land in a broader context. If we regard the most important properties concerning filtering and buffering for groundwater recharge, soils must provide both coarser pore systems (because based on the Hagen Poiseuille law, the velocity of the transport depends on the fourth power of the pore radius) and an increasing retention time that requires finer pores, regulating the more enhanced buffering processes due to a retarded mass flow and diffusion.

Food and fiber production in turn requires sufficient chemical base saturation and accessibility as well as storage and flux of nutrients and water, and optimal thermal conditions in combination with a large soil volume suitable for root penetration. Thus, a well-balanced proportion of water, gas and ions in combination with a perfect accessibility of surfaces and volumes is both essential for optimal plant growth, and a defined strength compete with external forces like mechanical impacts or air pollution.

Irrespective of various land-use management scenarios, the rigidity of systems (defined as transition from elastic to plastic properties and function changes) is the key parameter, but it is mostly ignored, although these limits affect

Received February 13, 2020

Correspondence: rhorn@soils.uni-kiel.de

(c) The Author(s) 2020. Published by Higher Education Press. This is an open access article under the CC BY license (http://creativecommons.org/licenses/by/4.0) 
not only the further soil genetic developments, but also the outcome of the more often applied modeling approaches.

Consideration of the well-known law of action and reaction allows us to describe the interaction between external (physical, mechanical and chemical) stress and the corresponding internal soil strength and will define the sensitivity of soils and their functions. Such considerations are essential if we intend to increase or at least maintain the soil functions like fluxes, root penetrability, filtering and buffering. Therefore, we need a paradigm shift in the general analytical approaches in two directions:

(1) Many decision processes or models are based on soil properties which derive from completely homogenized material like capacity parameters. Well-known parameters are cation exchange capacity, air capacity and plant available water. However, such data will not define or predict any in situ soil characteristics, because neither the accessibility of particle or pore wall surfaces nor the connectivity between pores within structured soils are considered. In undisturbed soils, properties and functions should be described by intensity parameters like cation exchange intensity, redox reactions, air permeability, gas diffusion, hydraulic and thermal conductivity and actual soil strength.

(2) Resilience is defined by the mechanical strength (the precompression stress) as the result of long-term geological, pedo and anthropogenic processes, and serves as the rigidity boundary. It distinguishes between the recompression stress (i.e., elastic and rigid properties) and the virgin compression stress range, where plastic deformation including irreversible changes of properties and functions occur (Fig. 1). As long as the actual soil structure remains rigid, all soil functions remain constant and are defined, which also guarantees stable corresponding ecological functions (Fig.2). With increasing soil aggregate formation (from homogeneous via prismatic to subangular blocky and finally crumbly structure) is the rigidity as well as the accessibility of particle and pore surfaces also enhanced and guarantees constant site productivity; furthermore, the biodiversity will be maintained. Anthropogenic processes like soil compaction and deformation result instead in a platy structure which is in fact very rigid but inaccessible for water, ions, gas, or roots and also changes the flux directions especially in even only slightly slopy regions more to horizontal downhill flow (Fig.2). Thus, all changes in the hydraulic or pneumatic functions like hydraulic or air conductivity, pore size distribution occur within the virgin compression stress range (Fig. 3). The same is the case for redox reactions, changes in biological activity (e.g., respiration) and microbial composition in soils in addition to the reduction of the carbon sequestration potential beyond the precompression stress value. The latter can be explained by the reduced accessibility of surfaces and affected physicochemical reactions.

If we do not consider these limits and properties, but consider soils as completely rigid systems, modeling approaches will be affected by a large uncertainty, because intense and not defined changes occur at soil conditions within the virgin compression load range. They will cause a reduced accessibility, availability and pore continuity, and

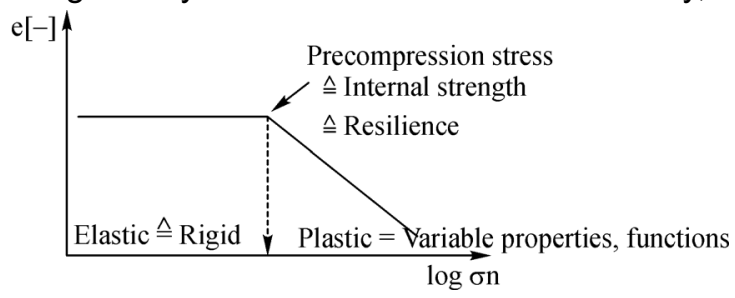

Fig. 1 Stress strain relation and the consequences for elastic or plastic soil behavior $(e=$ void ratio, $\sigma n=$ stress $)$. The precompression stress defines the internal soil strength and the resilience limit.

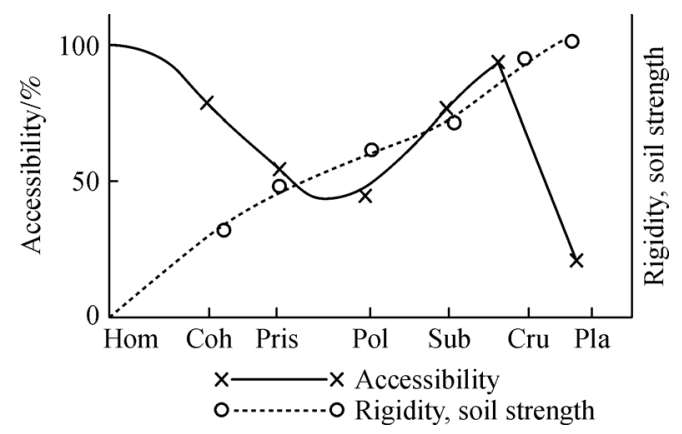

Fig. 2 Effect of aggregate type on the accessibility of surfaces (particle or pore walls) as well as the consequences for soil strength or rigidity. Hom $=$ Homogenous, $\mathrm{Coh}=$ Coherent, Pris $=$ Prismatic, $\mathrm{Pol}=$ Polyhedral, $\mathrm{Sub}=$ Subangular blocky, Cru $=\mathrm{Crumbly}, \mathrm{Pla}=\mathrm{Platy}$ structure. 


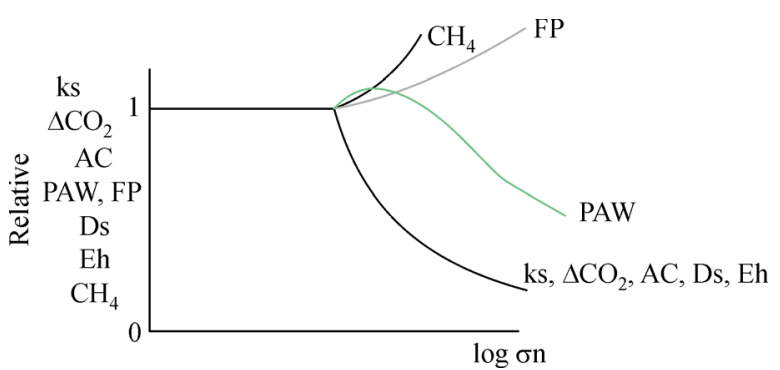

Fig. 3 Relative changes of soil properties within the virgin compression = plastic range. During the recompression load range remain the properties and functions mostly unchanged while in virgin compression load range induce increasing stresses applied $(\sigma n=$ stress $)$ changes in: $\mathrm{AC}=$ Air capacity, $\mathrm{CH}_{4}=$ Methane concentration, $\mathrm{CO}_{2}=$ Carbon dioxide, $\mathrm{Eh}=$ Redox potential, $\mathrm{FP}=$ Fine pores, $\mathrm{Ds}=\mathrm{Gas}$ diffusion, $\mathrm{ks}=$ Saturated hydraulic conductivity, and PAW $=$ Plant available water.

simultaneously increased pore tortuosity, changing three-dimensional fluxes and stress propagation or declined pore connectivity.

Thus, the main parameters and functions of the hydraulic, gas or heat flux under given potential gradients must be added by the rigidity information in order to finally obtain a more complete insight in the effect of land-use management systems on coupled hydraulic mechanical soil processes, defining the climate-food-energy-water nexus.

However, there have been no modeling attempts which differentiate between the rigid soil properties and the varying ones, nor are the boundaries defined for the in situ conditions worldwide. In order to predict side properties and functions reliably, the input parameters for modeling need to be limited to this recompression stress range, where the rigidity assumption still holds true, while further technical developments beyond the present ones are not to be forecasted within the virgin, i.e., plastic, deformation range.

In this context, we also need to regard the climate change induced alteration of temperature and rainfall amount and intensities. The expected higher temperature during the summer period and the resulting higher evapotranspiration will presumably also result in a more intense drying of deeper soil volumes, which coincide with cracking, altered functions and strength. Consequently, the structural shrinkage range will be extended, too, with a feedback loop to an increased precompression stress. However, the actual extend of these changes is neither quantified nor included as altered data part of the modeling approaches for future soil developments.

Therefore, we can define three major final issues for future research, which need to be linked as scenarios for any resulting political actions in order to strive for production of sufficient food, fiber and water on a long-term basis.

(1) Definition of the actual resilience limit defined, e.g., as precompression stress or soil strength, as the transition limit from elastic to plastic changes in soil functions in order to maintain the actual productivity and functionality for the future, because the reestablishment of former by better soil functions and properties requires too much time and energy worldwide.

(2) Definition of site-specific functionality maps which define specific land use or management forms, in order to maintain or optimize yield, soil protection and sustainable land-use management considering the limited site-specific resilience. Furthermore, the coupling of physical, mechanical, chemical, and biological processes is mandatory in order to obtain a more complete picture of in situ conditions as the basis for the quantification of the boundary conditions for a sustainable and long-term land-use management.

(3) Quantitative evaluation of climate change effects on soil properties, functions (hydraulic, thermal and gas fluxes) and strength, including an altered accessibility and availability of surfaces in order to provide reliable input parameters for uncounted prediction models.

The above-mentioned future research activities and political topics will be only successful and prospective, if the actual in situ soil properties and functions are clearly quantified and adjusted to the actual land use and climatic conditions. These properties are considered as the main boundaries for a sufficient plant growth, filtering and buffering also under altered climatic conditions. Together with the rigidity or strength boundaries is the quantification of the climate change effects irrespective of all existing scenarios the most important challenge to guarantee longterm safeguarding of local or worldwide supply facilities for the resident population also in the coming centuries.

\section{Reference}

1. Blum W, Eswaran H. Soils for sustaining global food production. Journal of Food Science, 2004, 69(2): 37-42 